\title{
The apple MdCOP1-interacting protein 1 negatively regulates hypocotyl elongation and anthocyanin biosynthesis
}

\author{
Hui Kang ${ }^{1}$, Ting-Ting Zhang ${ }^{2}$, Lu-Lu Fu², Yu-Xin Yao², Chun-Xiang You ${ }^{2}$ Xiao-Fei Wang ${ }^{2 *}$ and Yu-Jin Hao ${ }^{2 *}$
}

\begin{abstract}
Background: In plants, CONSTITUTIVELY PHOTOMORPHOGENIC 1 (COP1) is a key negative regulator in photoperiod response. However, the biological function of COP1-interacting protein 1 (CIP1) and the regulatory mechanism of the CIP1-COP1 interaction are not fully understood.

Results: Here, we identified the apple MdCIP1 gene based on the Arabidopsis AtCIP1 gene. Expression pattern analysis showed that MdCIP1 was constitutively expressed in various tissues of apple, and responded to stress and hormone signals at the transcriptional level. Ectopic expression of MdCIP1 complemented the phenotypes of the Arabidopsis cip1 mutant, and MdCIP1 inhibited anthocyanin biosynthesis in apple calli. In addition, the biochemical assay demonstrated that MdCIP1 could interact with MdCOP1 protein by their coiled-coil domain, and MdCIP1-OXI cop1-4 had a similar phenotype in photomorphogenesis with the cop1-4 mutant, suggesting that COP1 is epistatic to CIP1. Furthermore, the transient transformation assay indicated that MdCIP1 repressed anthocyanin biosynthesis in an MdCOP1-mediated pathway.
\end{abstract}

Conclusion: Take together, this study finds that MdCIP1 acts as a repressor in regulating hypocotyl elongation and anthocyanin biosynthesis through MdCOP1 in apple.

Keywords: CIP1, COP1, Apple, Hypocotyl, Anthocyanin

\section{Background}

From seed germination to seedling development, plants are precisely regulated by various environmental factors. Light is one of the most important environmental factors affecting plant growth, development and reproduction. Plants convert light energy into chemical energy through photosynthesis and store it in plants. They can also sense light signals through the photoreceptor, and then regulate the entire life cycle of plant growth and development [1, 2]. Plants mainly rely on three kinds of photoreceptors to sense

\footnotetext{
* Correspondence: xfwang2004@163.com; haoyujin@sdau.edu.cn ${ }^{2}$ State Key Laboratory of Crop Biology, Shandong Collaborative Innovation Center for Fruit and Vegetable Production with High Quality and Efficiency, College of Horticulture Science and Engineering, Shandong Agricultural University, Tai-An 271018, Shandong, China

Full list of author information is available at the end of the article
}

sunlight and transmit light signals, namely phytochromes (red and far-red light receptors), cryptochromes (blue light receptor) and UVR8 (UV-B light receptor) [3-5].

After being activated by light, photoreceptors enter the nucleus and interact directly with a series of factors to transmit light signals, thus changing the transcription pattern of plants, and finally leading to photomorphogenesis $[6,7]$. Photomorphogenesis is essential for seedling development. When the seeds germinate in the soil, the seedlings accelerate hypocotyl elongation to emerge from the soil. After reaching the light, hypocotyl elongation is inhibited while cotyledons quickly expand [8]. In addition, the biosynthesis of chlorophyll and anthocyanin is also a typical feature of photomorphogenesis $[8$, 9]. In the process of light signal transduction, CONSTITUTIVELY PHOTOMORPHOGENIC 1 (COP1) is the

\section{$\triangle B M C$}

(c) The Author(s). 2021 Open Access This article is licensed under a Creative Commons Attribution 4.0 International License, which permits use, sharing, adaptation, distribution and reproduction in any medium or format, as long as you give appropriate credit to the original author(s) and the source, provide a link to the Creative Commons licence, and indicate if changes were made. The images or other third party material in this article are included in the article's Creative Commons. licence, unless indicated otherwise in a credit line to the material. If material is not included in the article's Creative Commons licence and your intended use is not permitted by statutory regulation or exceeds the permitted use, you will need to obtain permission directly from the copyright holder. To view a copy of this licence, visit http://creativecommons.org/licenses/by/4.0/ The Creative Commons Public Domain Dedication waiver (http://creativecommons.org/publicdomain/zero/1.0/) applies to the data made available in this article, unless otherwise stated in a credit line to the data. 
most important negative factor, which is located downstream of photoreceptors [10]. COP1 is a conserved RING type E3 ubiquitin ligase, which participates in many physiological processes of different organisms, including plant growth and development, mammalian cell growth and metabolism [11].

In plants, COP1 is transported into the nucleus under dark conditions, thus regulates the seedlings to skotomorphogenesis by degrading the photomorphogenesis promoters LONG HYPOCOTYL IN FAR-RED 1 (HFR1), LONG AFTER FAR-RED LIGHT 1 (LAF1) and ELONGATED HYPOCOTYL 5 (HY5); under light conditions, COP1 is located in the cytoplasm, thus promotes biosynthesis of the downstream substrate, which promotes photomorphogenesis [12-14]. In addition, COP1 is involved in plant flowering, stomatal opening and closing, biological rhythm and fruit development [15-17]. Moreover, COP1 also participates in plant defense response, hormone signaling and miRNA biosynthesis [18-21].

COP1 protein contains an $\mathrm{N}$-terminal RING finger motif, followed by a coiled-coil domain and WD40 repeats, and several COP1-interacting proteins (CIPs) have been isolated in Arabidopsis [22-28]. Among them, CIP4 and CIP7 interact with coiled-coil domain of COP1, which are located in the nucleus and have transcriptional activation activity. CIP4 and CIP7 are also positive regulators of photomorphogenesis and potential direct downstream targets of COP1 [25, 27]. However, the functions of CIP4 and CIP7 are different. The decrease of CIP7 expression resulted in the decrease of light-dependent anthocyanin and chlorophyll biosynthesis, and did not affect the hypocotyl elongation. While the decrease of CIP4 expression inhibited hypocotyl elongation and chlorophyll content, and did not affect anthocyanin biosynthesis [25, 27]. CIP8 is a RING-H2 ligase, which can interact with the COP1 protein. CIP8 can also degrade HY5 through a ubiquitin ligase E2-dependent manner in vitro, but its biological function is not yet clear $[26,28]$.

Previous studies have found that CIP1 interacts with the coiled-coil domain of COP1 in vitro [24]. But the biological function of CIP1 and the biological significance of the CIP1-COP1 interaction are not fully understood. Here, we found that the apple homologous gene MdCIP1 negatively regulates photomorphogenesis. Moreover, MdCIP1 interacts with MdCOP1 and inhibits apple anthocyanin biosynthesis in an MdCOP1-mediated pathway.

\section{Results}

Protein structure of MdCIP1

The apple MdCIP1 (MDP0000230486) gene was blasted from the database Apple Genome V1.0 Predicted Peptides by using the protein sequence of Arabidopsis
AtCIP1 (AT5G41790). After SMART analysis of protein secondary structure (http://smart.embl-heidelberg.de/ smart/set_mode.cgi?NORMAL=1), MdCIP1 contains a potential coiled-coil domain, corresponding to aa 251684 of the full length MdCIP1, which is analogous to AtCIP1 (Fig. S1b). Then, Pymol program and VMD software were used to predict the 3D structure of MdCIP1 and AtCIP1. Surprisingly, the 3D structure of MdCIP1 and AtCIP1 is highly similar, although their protein sequence similarity is only $30 \%$ (Fig. S1a, c).

\section{Expression patterns of MdCIP1}

Through Gene Structure Display Server 2.0 analysis, it was found that the $M d C I P 1$ gene contains five exons, four introns and encodes 1640 amino acids (Fig. 1a). In order to analyze the tissue-specific expression of $M d C I P 1$, the transcription level of MdCIP1 in root, shoot, leaf, flower and fruit was detected. It was found that the expression level of $M d C I P 1$ was higher in leaves and lower in fruits (Fig. 1b). The response of MdCIP1 to light and dark conditions was also analyzed. After $1 \mathrm{~h}$ of light treatment, the expression of MdCIP1 increased rapidly. With the prolongation of the illumination time, the expression of $M d C I P 1$ did not change significantly. In dark treatment, there was no significant difference in expression level of $M d C I P 1$ at different time (Fig. 1c). The response of MdCIP1 to stress and hormone signals was also examined. As a result, in the early stage of salt and PEG treatment, the MdCIPlexpression was significantly up-regulated, reached the maximum after $12 \mathrm{~h}$ treatment, and then decreased (Fig. 1d, e). The response of MdCIP1 to ABA is faster than salt and PEG treatment, reaching the maximum after $6 \mathrm{~h}$ treatment (Fig. 1f). These results indicate that $M d C I P 1$ can respond to stress and hormone signals at the transcriptional level.

\section{MdCIP1 inhibits photomorphogenesis}

To investigate the function of CIP1 in photomorphogenesis, the cip1 mutant, $M d C I P 1$ heterologous overexpression lines and their hybrid Arabidopsis materials were obtained. These materials were identified at the DNA level (Fig. S2). The hypocotyl length of seedlings in light and darkness was quantified. As a result, the hypocotyl of the cip1 mutant was shorter than that of wild type under light and dark conditions (Fig. 2) [29]. MdCIP1 overexpression in the cip1 mutant complemented the phenotype of the cip1 mutant (Fig. 2). This result suggests that AtCIP1 and MdCIP1 play an inhibitory role in photomorphogenesis and are conserved in function. To further understand the function of MdCIP1 in apple, the MdCIP1-OX and MdCIP1-anti transgenic apple calli were constructed (Fig. 3b). The calli anthocyanin biosynthesis results show that compared with WT, MdCIP1OX accumulated less anthocyanin, and MdCIP1-anti 


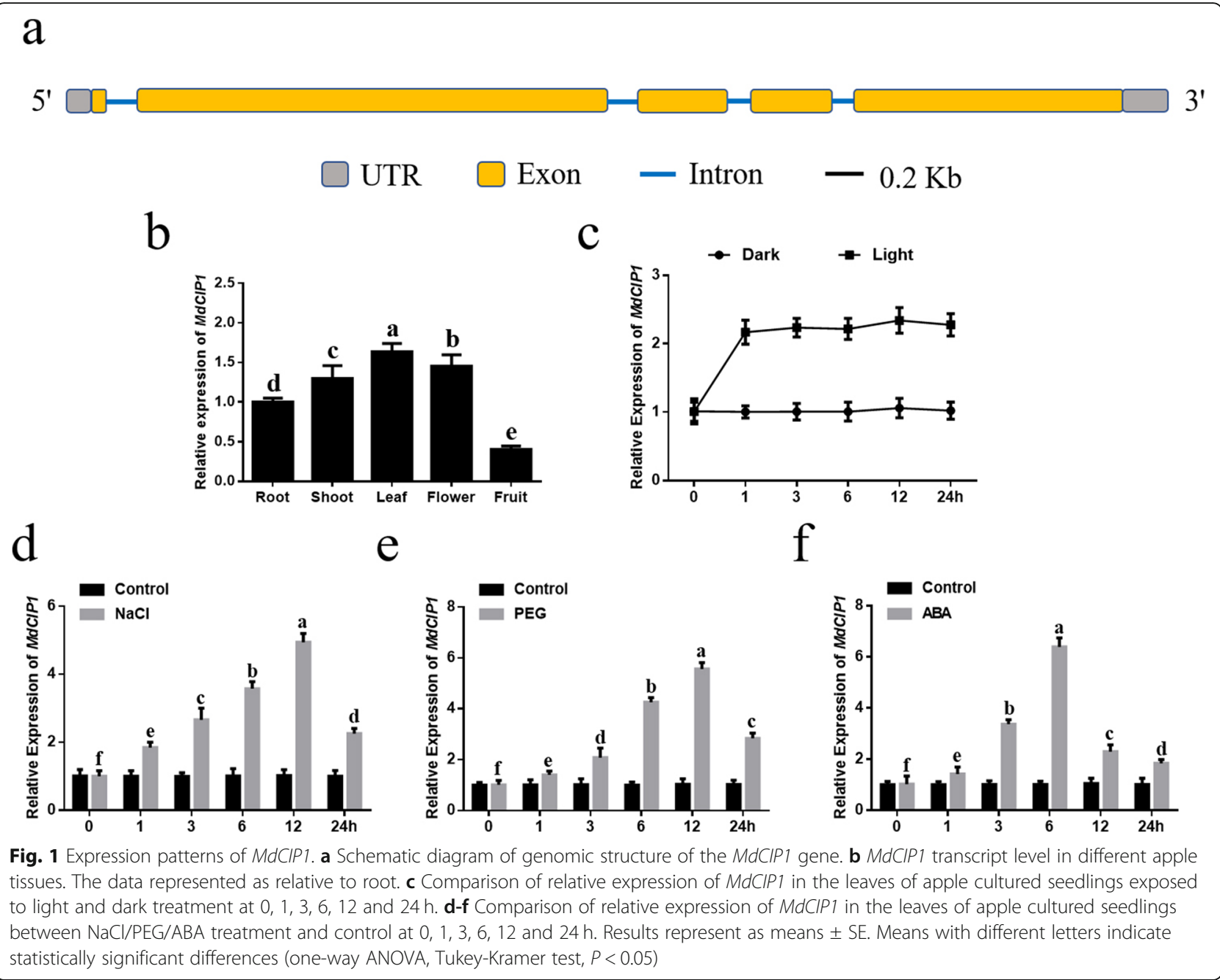

accumulated obviously more (Fig. 3a, c). This suggests that MdCIP1 represses anthocyanin biosynthesis in apple calli.

\section{MdCIP1 interacts with MdCOP1}

AtCIP1 interacts with AtCOP1 protein by their coiledcoil domains [24]. It is speculated that MdCIP1 interacts with $\mathrm{MdCOP} 1$, and was verified by $\mathrm{Y} 2 \mathrm{H}$ experiment. According to the protein domain analysis, MdCIP1 is divided into $\mathrm{N}$-terminal, a coiled-coil domain and $\mathrm{C}$ terminal; MdCOP1 contains a RING motif, a coiled-coil domain and WD40 repeats (Fig. 4a, b). Y2H results show that MdCIP1 also interacts with the coiled-coil domain of MdCOP1 via its coiled-coil domain (Fig. 4c). Moreover, the pull-down assay was also used to verify this interaction. The results showed that His-MdCOP1 protein was pulled down only by GST-MdCIP1 CC protein but not GST protein, demonstrating that the coiled-coil domain of MdCIP1 physically interacts with MdCOP1 in vitro (Fig. S3). MdCIP1 also interacts with AtCOP1 (Fig. S4), which is similar to that of AtCIP1-AtCOP1.

COP1 is epistatic to CIP1

In order to test whether CIP1 is dependent on COP1 in photomorphogenesis, the cop1-4 mutant, MdCIP1-OX and MdCIP1-OX/cop1-4 were obtained and proven by the DNA detection (Fig. S5). Interestingly, ectopic overexpression of $M d C I P 1$ in the cop1-4 mutant showed a similar phenotype of hypocotyl with that of $\operatorname{cop} 1-4$, no matter the light or dark treatments (Fig. 5). At the adult stage, MdCIP1-OX/cop1-4 also fully exhibited the features of cop1-4, dwarfing and branching (Fig. S6). This result indicates that COP1 is epistatic to CIP1.

\section{MdCIP1 represses anthocyanin biosynthesis in an MdCOP1-dependent pathway}

The roles of MdCIP1 and MdCOP1 in anthocyanin biosynthesis were also observed by transient infection of leaves. Empty vector served as a control. As a result, the 


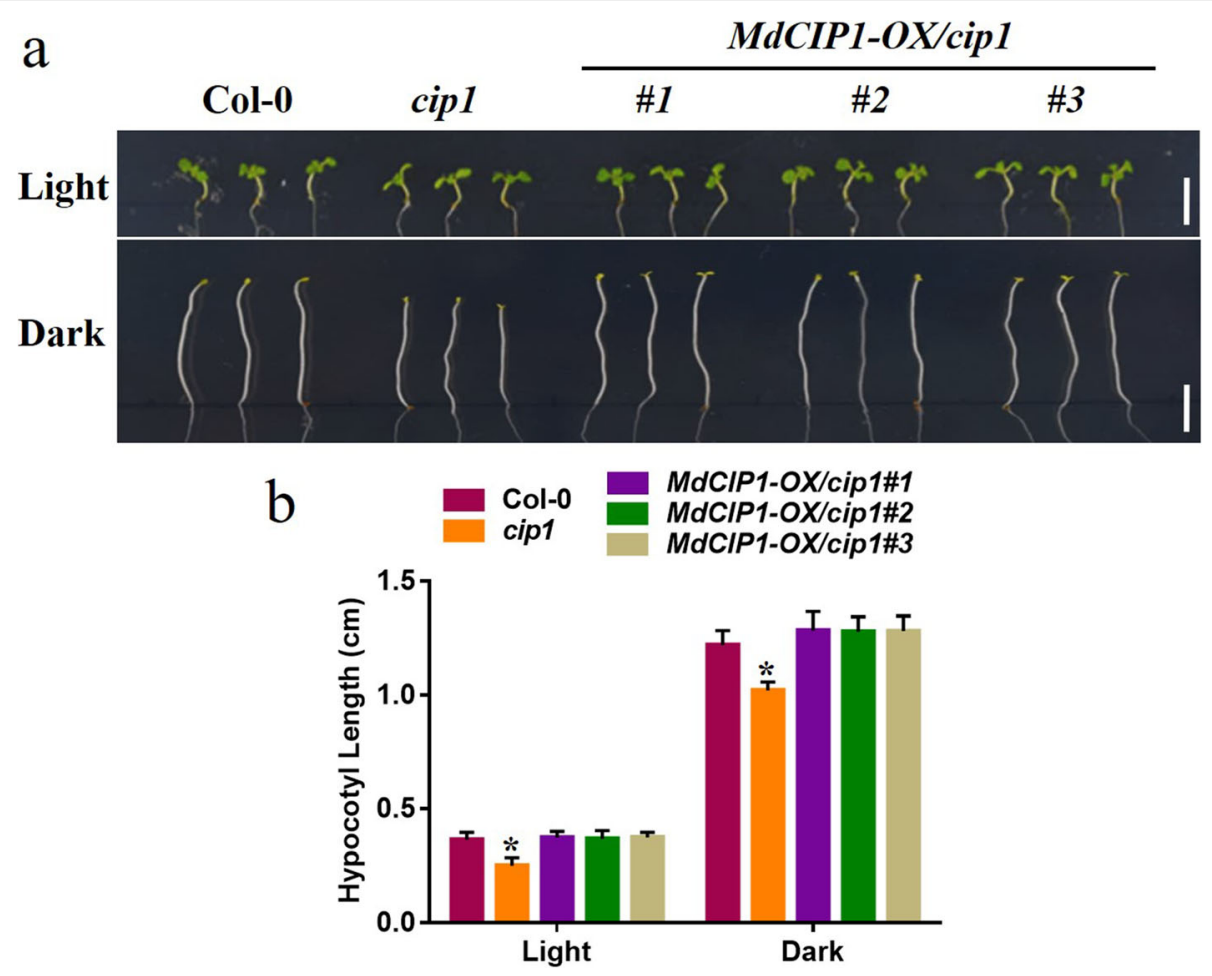

Fig. 2 MdCIP1 inhibits photomorphogenesis. a-b Representative picture and measurement of the hypocotyl of col-0, the cip1 mutant and MdCIP1-OX_ip 1\#1/2/3 Arabidopsis seedlings under the dark and light. Three independent biological replicates were performed. Results represent as means $\pm \mathrm{SE}(n=20)$. Asterisks indicate significantly different values $\left({ }^{*} \mathrm{P}<0.05\right)$. Bar $=0.5 \mathrm{~cm}$

MdCIP1 expression was up-regulated and anthocyanin decreased after MdCIP1-OX infection; the MdCOP1 expression was down-regulated and anthocyanin increased after MdCOP1-anti infection (Fig. 6a-d). When mixed infection, the anthocyanin content of MdCIP1-OX/ MdCOP1-anti was equal to that of MdCOP1-anti (Fig. 6ad). This indicates that MdCIP1 represses anthocyanin biosynthesis through MdCOP1 in apple leaves.

\section{Discussion}

'In Arabidopsis, CIP1 encodes 1586 amino acids and its relative molecular weight is up to $182.02 \mathrm{kDa}$ [24]. MdCIP1 protein contains 1640 amino acids with a relative molecular weight of $182.92 \mathrm{kDa}$. Although the protein sequence similarity of MdCIP1 and AtCIP1 is only $30 \%$, their 3D structures were remarkably similar (Fig. $\mathrm{S} 1$ ). This indicates that the amino acid sequence of CIP1 protein varies greatly with evolution among different species, but the key amino acids that maintain the protein structure remain unchanged. Structure determines function, which further indicates that CIP1 protein may have a conservative function during evolution. Figure 2 showed that both MdCIP1 and AtCIP1 restrain photomorphogenesis, which may prove this point.
MdCIP1-OX/cop1-4 showed a similar phenotype with that of cop1-4, indicating that CIP1 is located upstream of COP1 (Fig. 5; Fig. S6). CIP1 could interact with COP1 protein by their coiled-coil regions [24]. It is suggested that CIP1 may regulate COP1 at the protein level. COP1 has three domains: RING domain is responsible for recruiting ubiquitinbinding enzyme E2, the coiled-coil domain for dimerization and COP1-SPA interaction to form COP1-SPA complexes, and WD40 repeat region for interacting with substrates $[11,30]$. SPAs interact with COP1 and enhance the E3 ubiquitin ligase activity of COP1 [31-33]. Recently, a novel COP1 interacting protein, COP1 SUPPRESSOR 2 (CSU2) was identified [34]. CSU2 is located in the nucleus and could interact with COP1 through their coiled-coil regions. CSU2 represses COP1 E3 ubiquitin ligase activity by potentially interrupting dimerization of COP1 or COP1-SPA interaction [34]. However, CIP1, as a cytoskeletonassociated protein, exhibits a fibrillar pattern in the cytoplasm in hypocotyl cells [24]. Recent studies have shown that COP1 in the cytoplasm ubiquitously degrades the microtubule-associated protein WAVE-DAMPENED 2LIKE 3 (WDL3) under darkness in hypocotyl cells [35, 36]. It is speculated that CIP1 may be involved in the 


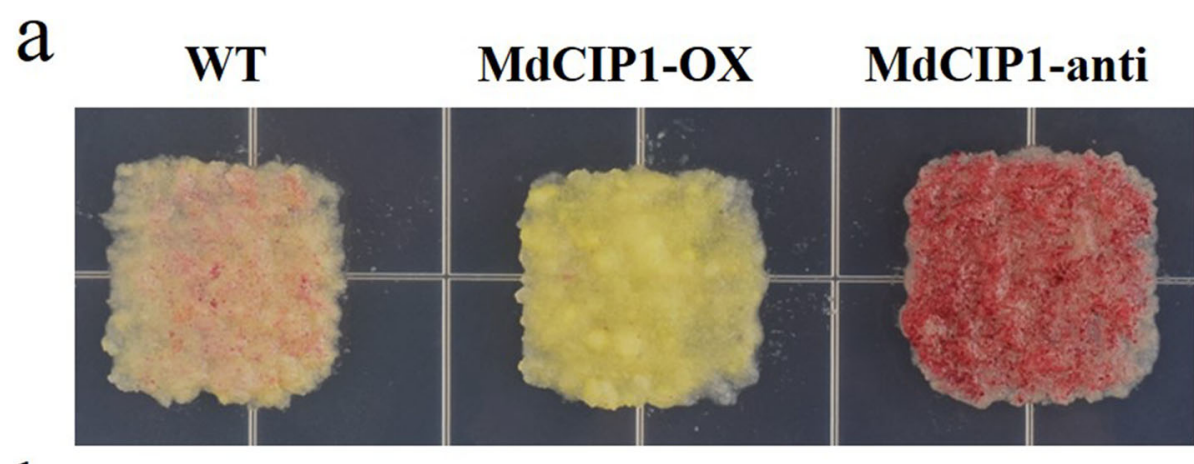

b
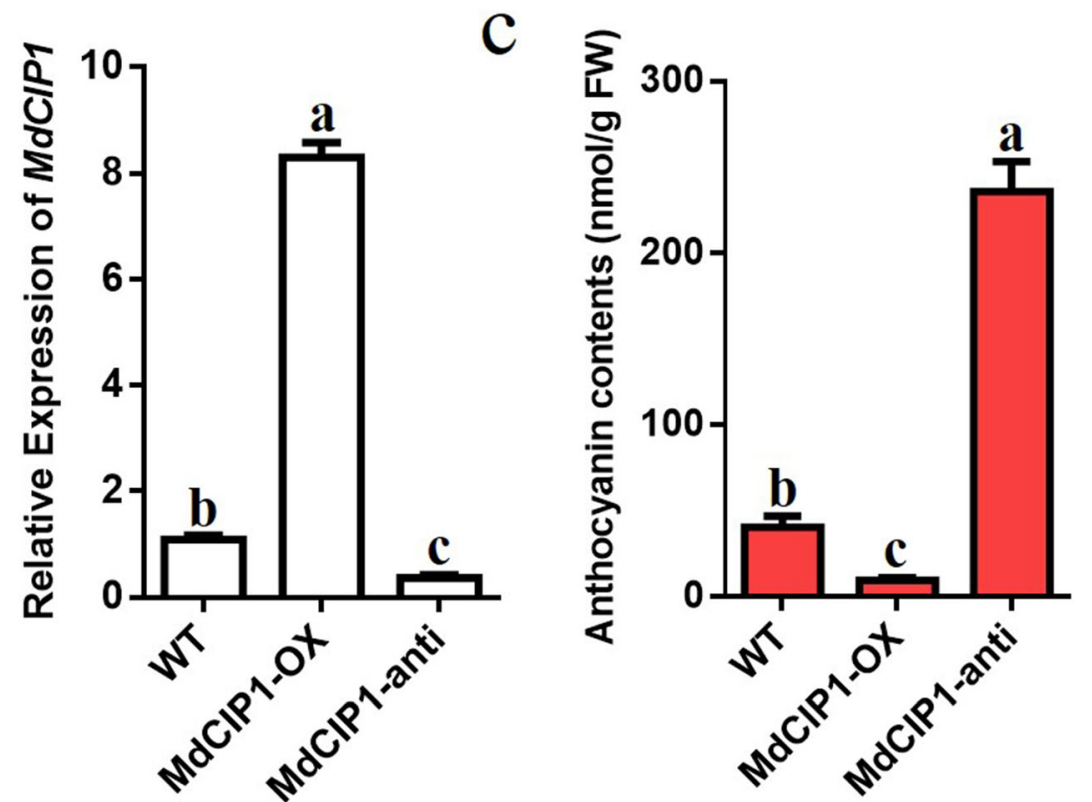

Fig. 3 MdCIP1 represses anthocyanin biosynthesis in apple calli. a Anthocyanin phenotype of the transgenic MdCIP1-OX and MdCIP1-anti calli. b The relative expression level of MdCIP1 in the transgenic MdCIP1-OX and MdCIP1-anti calli. c Anthocyanin content of the transgenic MdCIP1-OX and MdCIP1-anti calli. Results represent as means \pm SE, from three independent biological replicates. Means with different letters indicate statistically significant differences (one-way ANOVA, Tukey-Kramer test, $\mathrm{P}<0.05$ )

degradation of WDL3 by regulating COP1 protein in the cytoplasm, which is different from CSU2.

For apples, anthocyanin is important, which not only determines the color quality to a large extent, but also has the effect of antioxidant and scavenging reactive oxygen [37,38]. Anthocyanins, a class of key secondary metabolites, are synthesized by a series of enzymes (PAL, CHS, CHI, F3H, DFR, ANS and UFGT) encoded by structural genes and regulated by the MYB-bHLHWD40 transcription complex $[39,40]$. Anthocyanin biosynthesis is affected by environmental and hormonal signals [38, 41]. MdMYB1 and its alleles (MdMYB10 and MdMYBA) promotes anthocyanin biosynthesis by activating the transcription of structural genes $M d D F R$ and MdUF3GT [42, 43]. Studies have found that MdBT2 accelerated the degradation of MdMYB1 through proteasome pathway, thereby negatively regulating nitrogen- deficient induced anthocyanin biosynthesis [44]. Meanwhile, ABA, wounding, drought stress, and high light also induce anthocyanin biosynthesis through the "MdBT2-Target protein-MdMYB1" module (Target protein: MdbZIP44, MdWRKY40, MdERF38 and MdTCP46, respectively) [45-49]. MdCOP1 negatively regulate anthocyanin biosynthesis and the peel coloration of apple fruits by ubiquitination degradation of the MdMYB1 protein [50]. This study found that MdCIP1 repress anthocyanin biosynthesis, and MdCOP1 participates in this process (Fig. 6). The promoter activity of AtCIP1 can be induced by osmotic stress and ABA, and AtCIP1 is positively involved in ABA response [29]. MdCIP1 responds to hormonal and environmental signals at the transcriptional level (Fig. 1). It is presumed that the MdCIP1-MdCOP1-MdMYB1 pathway may also be used to reveal the regulation mechanism of 

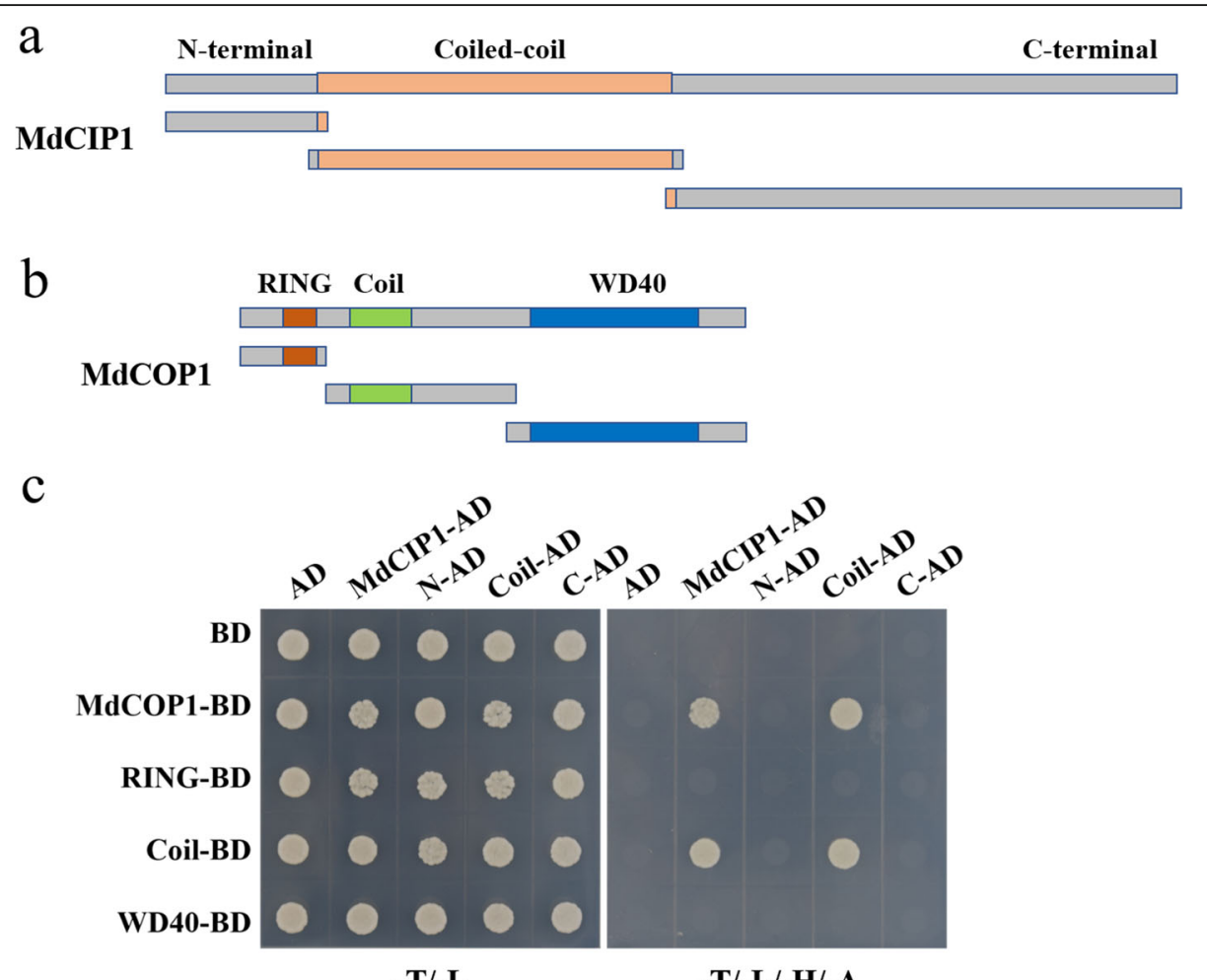

$-\mathbf{T} / \mathrm{L}$

$-\mathbf{T} /-\mathbf{L} /-\mathrm{H} /-\mathbf{A}$

Fig. 4 MdCIP1 interacts with MdCOP1 in Y2H assay. a Schematic diagram of MdCIP1 protein domains. b Schematic diagram of MdCOP1 protein domains. c Different truncated segments of MdCIP1 and MdCOP1 were connected to AD and BD vectors, respectively. MdCIP1 interacts with the coiled-coil domain of MdCOP1 via its coiled-coil domain

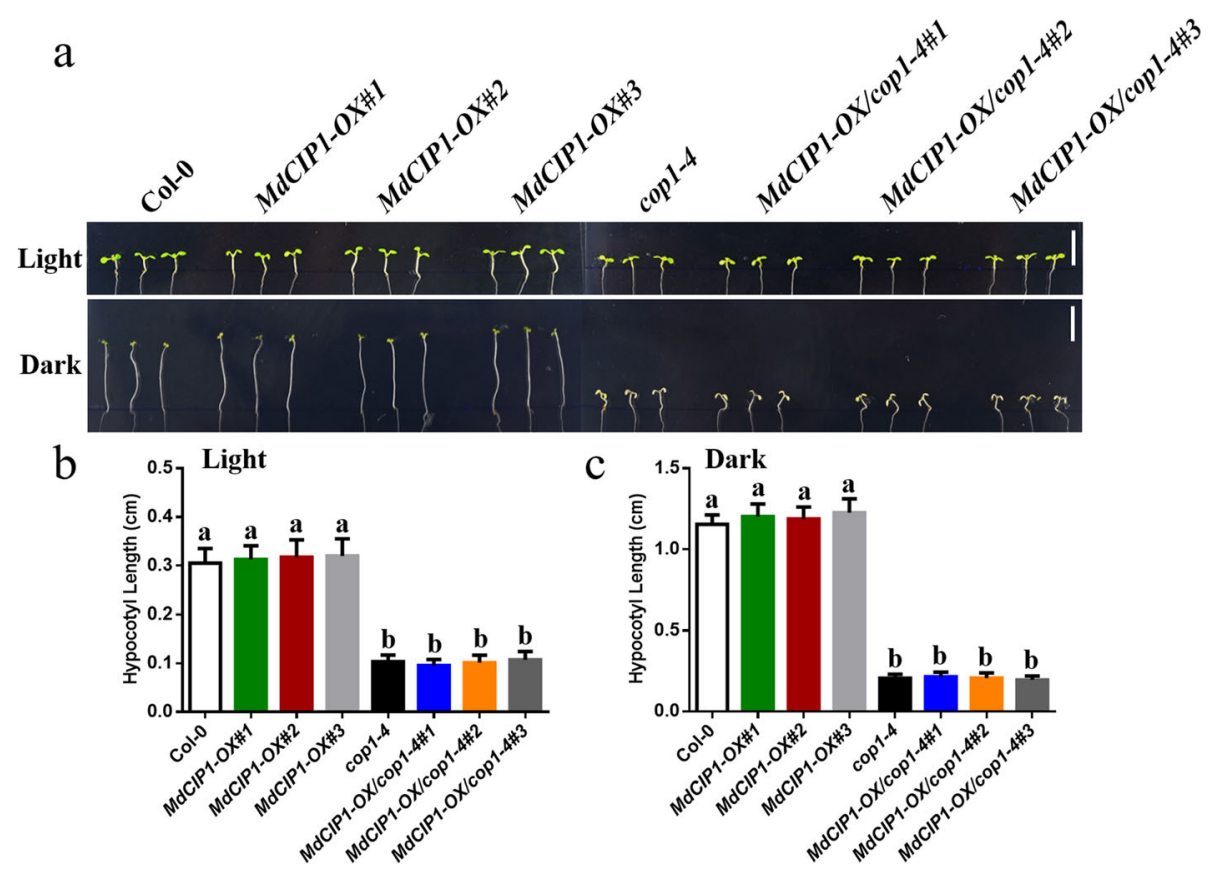

Fig. 5 COP1 is epistatic to CIP1. a-b Representative picture and measurement of the hypocotyl of col-0, MdCIP1-OX, cop1-4 and MdCIP1-OX/COP14 Arabidopsis seedlings under the dark and light. Three independent biological replicates were performed. Results represent as means \pm SE $(n=$ 20). Means with different letters indicate statistically significant differences (one-way ANOVA, Tukey-Kramer test, $P<0.05$ ). Bar $=0.5 \mathrm{~cm}$ 


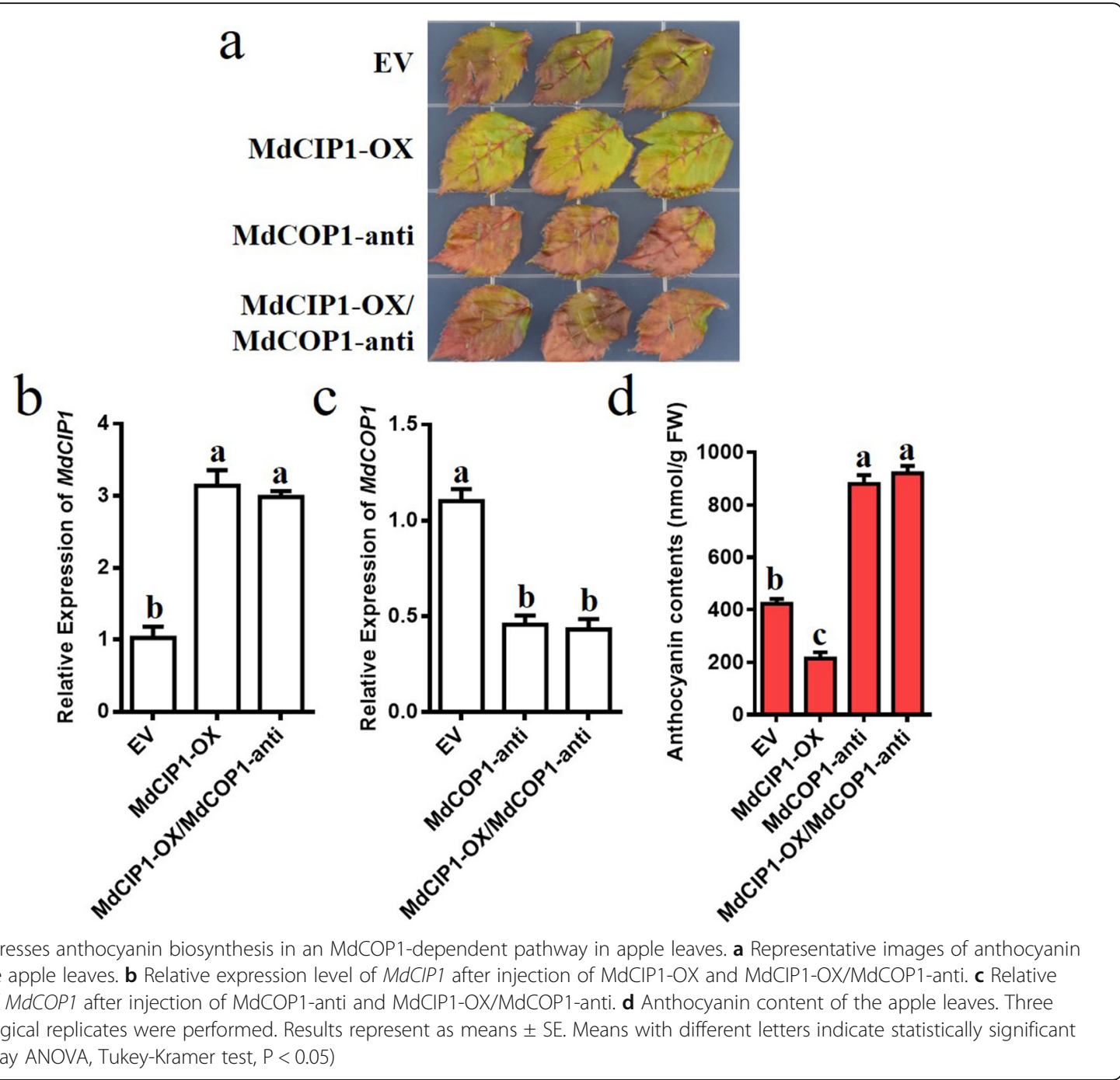

anthocyanin biosynthesis by hormonal and environmental signals, providing a new perspective on the hormonal and environmental impact on fruit coloration.

\section{Conclusion}

MdCIP1, upstream regulator of MdCOP1, plays an inhibitory role in hypocotyl elongation and anthocyanin biosynthesis by interacting with MdCOP1 in apple.

\section{Methods}

\section{Plant materials and growth conditions}

The 'Orin' apple calli was provided by Prof. Takaya Moriguchi of the National Institute of Fruit Tree Science, Japan, and has been widely used in peer laboratories [51,52]. The 'Orin' apple calli were cultured on MS medium with 0.4 $\mathrm{mg} / \mathrm{L}$ 6-BA, $1.5 \mathrm{mg} / \mathrm{L} 2,4-\mathrm{D}$ and $3 \%$ sucrose at $25^{\circ} \mathrm{C}$. The apple calli were subcultured at 3 weeks intervals under darkness.
A line with high regeneration capacity isolated from 'Royal Gala' named 'GL-3' has been widely used in peer laboratories $[53,54]$. We declare that the collection of plant materials complies with institutional, national, or international guidelines. The 'GL-3' apple culture seedling was provided by Prof. Zhi-Hong Zhang of Shenyang Agricultural University, China. The GL3 apple culture seedlings were cultured on MS medium with $0.5 \mathrm{mg} / \mathrm{L}$ 6-BA, $0.1 \mathrm{mg} / \mathrm{L} \mathrm{GA}_{3}, 0.2 \mathrm{mg} / \mathrm{L} \mathrm{NAA}$ and $3 \%$ sucrose under $16 \mathrm{~h}$ light $/ 8 \mathrm{~h}$ dark condition at $25^{\circ} \mathrm{C}$. The 5 -yearold 'GL-3' apple trees in the experimental station of Shandong Agricultural University were used for tissue expression analysis. For expression pattern analysis, onemonth-old apple culture seedlings were treated with light, darkness, $\mathrm{NaCl}$, PEG and ABA, respectively [55]. The culture seedlings were pretreated in the dark for one day, half of which were treated with light $(100 \mu \mathrm{mol} /$ $\mathrm{m}^{2} / \mathrm{s}$ ), and the other half treated with darkness. The culture seedlings were pretreated in liquid medium for one 
day, and then transferred to liquid medium containing $150 \mathrm{mmol} / \mathrm{L} \mathrm{NaCl}, 10 \%$ PEG6000 and $100 \mu \mathrm{mol} / \mathrm{L} \mathrm{ABA}$, respectively. The whole seedlings were sampled at 0,1 , $3,6,12$, and $24 \mathrm{~h}$ after treatment for subsequent analyses. Each treatment was performed with at least three independent biological repetitions.

Arabidopsis thaliana ecotype Columbia-0 (Col-0), was used as wild type, obtained from Arabidopsis Biological Resource Center (ABRC). The cip1 (Salk_070302) mutant was provided by Prof. Qing-Qiu Gong of Nankai University, China, which originally obtained from ABRC [29]. The cop1-4 mutant was provided by Prof. HongQuan Yang of Shanghai Normal University, China [23]. Surface-sterilized Arabidopsis seeds were sown on $1 / 2$ MS medium with $1 \%$ sucrose. The seeds were kept at $4{ }^{\circ} \mathrm{C}$ for $3 \mathrm{~d}$ in the dark, then transferred to a $16 \mathrm{~h}$-light period at $22^{\circ} \mathrm{C}$.

\section{Vector construction and plant transformation}

Leaves of 'GL-3' culture seedlings were used to clone the coding sequence of $M d C I P 1$ and $M d C O P 1$. Leaves of Col-0 seedlings were used to clone the coding sequence of AtCOP1. To get the transgenic lines, the coding sequence and antisense fragment of MdCIP1 or MdCOP1 were inserted into pRI101 vector by Sall and BamHI double digestion, and Agrobacterium tumefaciens strain GV3101 was used to transform apple calli and Arabidopsis, and 35S::MdCIP1-OX transgenic Arabidopsis, 35S:: MdCIP1-OX, 35S::MdCIP1-anti and 35S::MdCOP1-anti transgenic calli were obtained, respectively. Transformations of Arabidopsis and apple calli followed the methods described in $[56,57]$. The primers for vector construction are shown in Additional file 1: Table S1.

\section{RNA extraction and qRT-PCR analysis}

Total RNA of plant materials was extracted using RNA plant Plus Reagent Kit (CWBIO, China), and PrimeScript first-strand cDNA synthesis kit (Takara, Japan) was used to performed reverse transcription assay. The qRT-PCR was performed using the UltraSYBR Mixture (CWBIO, China) and ran on ABI Step One Plus system (ABI, USA). Md18S was used as the reference gene. The qRT-PCR program was performed under the following condition (40 cycles): $95^{\circ} \mathrm{C}$ for $15 \mathrm{~s}$ and $60^{\circ} \mathrm{C}$ for $40 \mathrm{~s}$. The qRT-PCR assay was carried out by three independent biological and three technical replicates. $2^{-\Delta \Delta C t}$ method was applied to calculate relative transcript level [58]. The specific primers are listed in Additional file 1: Table S1.

Protein sequence alignment and 3D structure prediction Protein sequence alignment was performed by the DNAM AN software. Protein 3D structure was predicted by Phyre ${ }^{2}$ (http://www.sbg.bio.ic.ac.uk/phyre2/html/page.cgi?id= index), and composite was obtained using the Pymol program v6.5.1. Root-mean-square deviation (RMSD) values were calculated by VMD software v1.2.1 to reflect the match [59]. The genomic sequence of the $M d C I P 1$ gene was selected and downloaded from Phytozome v12.1 (https://phytozome.jgi.doe.gov/pz/portal.html).

\section{Yeast two-hybrid (Y2H) assay}

Different truncated segments of MdCIP1 were connected to pGAD424 (AD) by EcoRI and BamHI double digestion. Different truncated segments of $M d C O P 1$ were connected to pGBT9 (BD) by EcoRI and BamHI double digestion. Different truncated segments of AtCOP1 were connected to pGBT9 (BD) by EcoRI and Sall double digestion. The $\mathrm{Y} 2 \mathrm{H}$ assay was performed according to the Yeast Transformation System 2 protocol (Clontech). The two plasmids were co-transformed into the $\mathrm{Y} 2 \mathrm{H}$ strain using the lithium acetate method and cultured at $28^{\circ} \mathrm{C}$. The yeast was grown on yeast selective medium lack of tryptophan and leucine $(-\mathrm{T} /-\mathrm{L})$ and then lack of tryptophan, leucine, histidine and Adenine $(-\mathrm{T} /-\mathrm{L} /-\mathrm{H} /-\mathrm{A})$ [60]. The primers are shown in Additional file 1: Table S1.

\section{Pull-down assay}

The full-length sequence of MdCOP1 was introduced into the pET-32a (His) by EcoRI and SalI double digestion. The coiled-coil domain of MdCIP1 was introduced into the pGEX-4 T-1 (GST) by BamHI and Sall double digestion. The recombinant plasmids were transformed into Escherichia coli BL21 (TransGen Biotech) to express His-MdCOP1 and GST-MdCIP1 CC protein, respectively. The pull-down assay was performed according to the instructions of the Pierce $^{\mathrm{Tw}}$ GST Spin Purification Kit (Thermo Fisher Scientific). Then samples were detected by western blotting with anti-His and anti-GST antibodies, respectively.

\section{Determination of the total anthocyanin content}

To accumulate anthocyanin, 15-day apple calli were grown on low-nitrogen $(0.2 \mathrm{mmol} / \mathrm{L}$ nitrate) medium under strong light supplemented with UV-B for $7 \mathrm{~d}$ at $17^{\circ} \mathrm{C}$. Approximately $0.1 \mathrm{~g}$ of the samples were soaked and incubated in 1 $\mathrm{ml}$ extraction buffer (95\% ethanol:1.5 M HCl $=85: 15$, v/v) overnight in the dark at room temperature. The absorbances were measured at 530, 620 and $650 \mathrm{~nm}$ using a spectrophotometer. $\mathrm{OD}_{\lambda}=\left(\mathrm{A}_{530}-\mathrm{A}_{620}\right)-0.1\left(\mathrm{~A}_{650}-\mathrm{A}_{620}\right)$. The anthocyanin content was quantified by the following formula: $\mathrm{OD}_{\lambda} / \xi_{\lambda} \times \mathrm{V} / \mathrm{m} \times 10^{6}\left(\mathrm{nmol} \times \mathrm{g}^{-1}\right.$ fresh weight; $\mathrm{V}$ : volume; m: weight; $\left.\xi_{\lambda}: 4.62 \times 10^{4}\right)$ [61].

\section{Measurement of the hypocotyl length}

The Arabidopsis material including Col-0, cip1, cop1-4, MdCIP1-OX/cip1\#1/2/3, MdCIP1-OX/cop1-4\#1/2/3 were used to quantify the length of hypocotyl. The seeds were kept 
at $4{ }^{\circ} \mathrm{C}$ for $3 \mathrm{~d}$ in the dark. Then the seeds were transferred to light ( $16 \mathrm{~h}$ light $/ 8 \mathrm{~h}$ dark) or dark conditions, and grown at $22^{\circ} \mathrm{C}$ for $5 \mathrm{~d}$. The ImageJ software was used to measure the length of hypocotyls. Approximately 20 hypocotyls were quantified for each material. The experiments were performed with at least three independent biological repetitions.

\section{Transient transformation in apple leaves}

The 35S::MdCIP1-OX (pRI101-MdCIP1), 35S::MdCOP1anti (pRI101-asMdCOP1) and EV (pRI101) plasmids were transformed into Agrobacterium tumefaciens strain GV3101. The leaves cut from the 20-day apple culture seedlings were incubated with Agrobacterium, and vacuumed twice for $20 \mathrm{~min}$. The transiently transformed apple leaves were treated on low-nitrogen $(0.2 \mathrm{mmol} / \mathrm{L} \mathrm{ni-}$ trate) medium under strong light supplemented with UV$\mathrm{B}$ for $3-5 \mathrm{~d}$ at $17^{\circ} \mathrm{C}$. The leaves were harvested for gene expression analysis and anthocyanin content determination [62]. Each experiment required about 60 leaves, and at least three independent biological repetitions were carried out.

\section{Supplementary Information}

The online version contains supplementary material available at https://doi. org/10.1186/s12870-020-02789-3.

Additional file 1: Table S1. Primers used in this study.

Additional file 2: Table S1-4. The data of hypocotyl length and anthocyanin content presented in this study.

Additional file 3. The coding and protein sequences of MdCIP1, MdCOP1, AtCIP1 and AtCOP1.

Additional file 4. The original gel/blot images presented in this study.

Additional file 5: Fig. S1. The protein structure comparison of MdCIP1 and AtCIP1. Fig. S2. Identification of the MdCIP1-OX/cip1 Arabidopsis seedlings at the DNA level. Fig. S3. The coiled-coil region of MdCIP1 interacts with MdCOP1 in vitro pull-down assay. Fig. S4. MdCIP1 interacts with AtCOP1. Fig. S5. Identification of the MdCIP1-OX/cop1-4 Arabidopsis seedlings at the DNA level. Fig. S6. MdCIP1-OX/cop1-4 presents the cop 1-4 phenotype at the adult stage.

\section{Abbreviations}

COP1: Constitutively photomorphogenic 1; LAF1: Long after far-red light 1; HY5: Elongated hypocotyl 5; HFR1: Long hypocotyl in far-red 1; CIP: COP1interacting protein; SPA: Suppressor of phya-105; WDL3: Wave-dampened 2like 3; CSU2: COP1 suppressor 2; PAL: Phenylalanine ammonialyase; $\mathrm{CHI}$ : Chalcone isomerase; CHS: Chalcone synthase; DFR: Dihydroflavonol 4reductase; F3H: Flavanone 3-hydroxylase; UFGT: Flavonoid 3-Oglucosyltransferase; ANS: Anthocyanidin synthase; Y2H: Yeast two-hybrid; SMART: Simple modular architecture research tool; ABRC: Arabidopsis biological resource center

\section{Acknowledgements}

We thank Prof. Qing-Qiu Gong of Nankai University, China, for providing the cip1 mutant. We thank Prof. Hong-Quan Yang of Shanghai Normal University, China, for providing the cop1-4 mutant. We thank Prof. Zhi-Hong Zhang of Shenyang Agricultural University, China, for providing the 'GL3' apple culture seedling. We thank Prof. Takaya Moriguchi of the National Institute of Fruit Tree Science, Japan, for providing the 'Orin' apple calli.

\section{Authors' contributions}

YJH, XFW and HK designed the research, analysed the data and wrote the manuscript. HK, TTZ and LLF preformed the experiments. YXY and CXY provided technical assistance. All authors have read and approved the manuscript.

\section{Funding}

This work was supported by grants from Shandong Province (ZR2018MC021 and J18KA174), the National Natural Science Foundation of China (U1706202), the open funds of the State Key Laboratory of Crop Genetics and Germplasm Enhancement (ZW202008), Ministry of Agriculture of China (CARS-27), and the National Key Research and Development Program (2018YFD1000200). The funder had no roles in conceiving, designing, or conducting this project.

\section{Availability of data and materials}

All the data about the present study has been included in the table and/or figure form in the current manuscript or additional files. The MdCIP1 (MDP0000230486) and MdCOP1 (MDP0000245133) sequences are available at apple genome database V1.0 (https://www.rosaceae.org/) [50]; the AtCIP1 (AT5G41790) and AtCOP1 (AT2G32950) sequences are available at Arabidopsis database (https://www.arabidopsis.org/).

Ethics approval and consent to participate

Not applicable.

\section{Consent for publication}

Not applicable.

\section{Competing interests}

The authors declare that they have no competing interests.

\section{Author details}

${ }^{1}$ State Key Laboratory of Crop Stress Biology for Arid Areas/Shaanxi Key Laboratory of Apple, College of Horticulture, Northwest A\&F University, Yang-Ling 712100, Shaanxi, China. ${ }^{2}$ State Key Laboratory of Crop Biology, Shandong Collaborative Innovation Center for Fruit and Vegetable Production with High Quality and Efficiency, College of Horticulture Science and Engineering, Shandong Agricultural University, Tai-An 271018, Shandong, China.

Received: 30 June 2020 Accepted: 8 December 2020

Published online: 06 January 2021

\section{References}

1. Wang HY, Deng XW. Dissecting the phytochrome A-dependent signaling network in higher plants. Trends Plant Sci. 2003;8(4):0-178.

2. Jiao $Y$, Lau OS, Deng $X W$. Light-regulated transcriptional networks in higher plants. Nat Rev Genet. 2007;8(3):217-30.

3. Lin C. Blue light receptors and signal transduction. Plant Cell. 2002; 14(Suppl):S207-25

4. Li J, Li G, Wang H, Wang DX. Phytochrome signaling mechanisms. Arabidopsis Book. 2011;9:e0148.

5. Rizzini L, Favory JJ, Cloix C, Faggionato D, O'Hara A, Kaiserli E, Baumeister R, Schäfer E, Nagy F, Jenkins GI, Ulm R. Perception of UV-B by the Arabidopsis UVR8 protein. Science. 2011;332(6025):103-6.

6. Huang $X$, Ouyang $X$, Deng XW. Beyond repression of photomorphogenesis: role switching of COP/DET/FUS in light signaling. Curr Opin Plant Biol. 2014; 21:96-103.

7. Podolec R, Ulm R. Photoreceptor-mediated regulation of the COP1/SPA E3 ubiquitin ligase. Curr Opin Plant Biol. 2018;45:18-25.

8. Sullivan JA, Deng XW. From seed to seed: the role of photoreceptors in Arabidopsis development. Dev Biol. 2003;260:289-97.

9. Kami C, Lorrain S, Hornitschek P, Fankhauser C. Light-regulated plant growth and development. Curr Top Dev Biol. 2010;91:29-66.

10. Lau OS, Deng XW. The photomorphogenic repressors COP1 and DET1: 20 years later. Trends Plant Sci. 2012;17:584-93.

11. Yi C, Deng XW. COP1-from plant photomorphogenesis to mammalian tumorigenesis. Trends Cell Biol. 2005;15(11):618-25. 
12. Osterlund MT, Hardtke CS, Wei N, Deng XW. Targeted destabilization of HY5 during light-regulated development of Arabidopsis. Nature. 2000;405(6785): 462-6.

13. Seo HS, Yang JY, Ishikawa M, Bolle C, Ballesteros ML, Chua NH. LAF1 ubiquitination by COP1 controls photomorphogenesis and is stimulated by SPA1. Nature. 2003:423(6943):995-9.

14. Jang IC, Yang JY, Seo HS, Chua NH. HFR1 is targeted by COP1 E3 ligase for post-translational proteolysis during phytochrome a signaling. Genes Dev. 2005;19(5):593-602.

15. Liu LJ, Zhang YC, Li QH, Sang Y, Mao J, Lian HL, Wang L, Yang HQ. COP1mediated ubiquitination of CONSTANS is implicated in cryptochrome regulation of flowering in Arabidopsis. Plant Cell. 2008;20(2):292-306.

16. Khanna R, Li J, Tseng TS, Schroeder JI, Ehrhardt DW, Briggs WR. COP1 jointly modulates cytoskeletal processes and electrophysiological responses required for stomatal closure. Mol Plant. 2014;7(9):1441-54.

17. Zoratti L, Karppinen K, Luengo Escobar A, Häggman H, Jaakola L. Lightcontrolled flavonoid biosynthesis in fruits. Front Plant Sci. 2014;5:534

18. Catalá R, Medina J, Salinas J. Integration of low temperature and light signaling during cold acclimation response in Arabidopsis. Proc Natl Acad Sci U S A. 2011;108(39):16475-80.

19. Cho SK, Chaabane SB, Shah P, Poulsen CP, Yang SW. COP1 E3 ligase protects HYL1 to retain microRNA biogenesis. Nat Commun. 2014;5:5867.

20. Kim JY, Jang IC, Seo HS. COP1 controls abiotic stress responses by modulating AtSIZ1 function through its E3 ubiquitin ligase activity. Front Plant Sci. 2016;7:1182.

21. Yu YW, Wang J, Shi H, Gu JT, Dong JG, Deng XW, Huang RF. Salt stress and ethylene antagonistically regulate nucleocytoplasmic partitioning of COP1 to control seed germination. Plant Physiol. 2016;170(4):2340-50.

22. Deng XW, Matsui M, Wei N, Wagner D, Chu AM, Feldmann KA, Quail PH. COP1, an Arabidopsis regulatory gene, encodes a protein with both a zincbinding motif and a G beta homologous domain. Cell. 1992;71(5):791-801.

23. McNellis TW, von Arnim AG, Araki T, Komeda Y, Miséra S, Deng XW. Genetic and molecular analysis of an allelic series of cop 1 mutants suggests functional roles for the multiple protein domains. Plant Cell. 1994;6(4):487-500.

24. Matsui M, Stoop CD, von Arnim AG, Wei N, Deng XW. Arabidopsis COP1 protein specifically interacts in vitro with a cytoskeleton-associated protein, CIP1. Proc Natl Acad Sci U S A. 1995;92(10):4239-43.

25. Yamamoto $Y Y$, Matsui $M$, Ang LH, Deng XW. Role of a COP1 interactive protein in mediating light-regulated gene expression in Arabidopsis. Plant Cell. 1998;10(7):1083-94.

26. Torii KU, Stoop-Myer CD, Okamoto H, Coleman JE, Matsui M, Deng XW. The RING finger motif of photomorphogenic repressor COP1 specifically interacts with the RING-H2 motif of a novel Arabidopsis protein. J Biol Chem. 1999;274(39):27674-81.

27. Yamamoto YY, Deng X, Matsui M. Cip4, a new COP1 target, is a nucleuslocalized positive regulator of Arabidopsis photomorphogenesis. Plant Cell. 2001;13(2):399-411.

28. Hardtke CS, Okamoto H, Stoop-Myer C, Deng XW. Biochemical evidence for ubiquitin ligase activity of the Arabidopsis COP1 interacting protein 8 (CIP8). Plant J. 2002;30(4):385-94.

29. Ren C, Zhu X, Zhang P, Gong Q. Arabidopsis COP1-interacting protein 1 is a positive regulator of ABA response. Biochem Biophys Res Commun. 2016; 477(4):847-53.

30. Holm M, Hardtke CS, Gaudet R, Deng XW. Identification of a structural motif that confers specific interaction with the WD40 repeat domain of Arabidopsis COP1. EMBO J. 2001;20(1-2):118-27.

31. Saijo $Y$, Sullivan JA, Wang $H$, Yang J, Shen Y, Rubio V, Ma L, Hoecker $H$, Deng XW. The COP1-SPA1 interaction defines a critical step in phytochrome A-mediated regulation of HY5 activity. Genes Dev. 2003;17:2642-7.

32. Zhu D, Maier A, Lee JH, Laubinger S, Saijo Y, Wang H, Qu LJ, Hoecker U, Deng XW. Biochemical characterization of Arabidopsis complexes containing CONSTITUTIVELY PHOTOMORPHOGENIC 1 and SUPPRESSOR OF PHYA proteins in light control of plant development. Plant Cell. 2008;20: 2307-23.

33. Sheerin DJ, Menon C, Zur Oven-Krockhaus S, Enderle B, Zhu L, Johnen P, Schleifenbaum F, Stierhof YD, Huq E, Hiltbrunner A. Light-activated phytochrome $a$ and $B$ interact with members of the SPA family to promote photomorphogenesis in Arabidopsis by reorganizing the COP1/SPA complex. Plant Cell. 2015;27:189-201.

34. Xu D, Lin F, Jiang Y, Ling J, Hettiarachchi C, Tellgren-Roth C, Holm M, Wei N, Deng XW. Arabidopsis COP1 SUPPRESSOR 2 represses COP1 E3 ubiquitin ligase activity through their coiled-coil domains association. PLoS Genet. 2015;11(12):e1005747.

35. Liu X, Qin T, Ma Q, Sun J, Liu Z, Yuan M, Mao T. Light-regulated hypocotyl elongation involves proteasome-dependent degradation of the microtubule regulatory protein WDL3 in Arabidopsis. Plant Cell. 2013;25(5):1740-55.

36. Lian N, Liu X, Wang X, Zhou Y, Li H, Li J, Mao T. COP1 mediates dark-specific degradation of microtubule-associated protein WDL3 in regulating Arabidopsis hypocotyl elongation. Proc Natl Acad Sci U S A. 2017;114(46):12321-6.

37. Horbowicz M, Kosson R, Grzesiuk A, Debski H. Anthocyanins of fruits and vegetables-their occurrence, analysis and role in human nutrition. Vegetable Crops Res Bull. 2008;68:5-22.

38. Jaakola L. New insights into the regulation of anthocyanin biosynthesis in fruits. Trends Plant Sci. 2013;18:477-83.

39. Hichri I, Barrieu F, Bogs J, Kappel C, Delrot S, Lauvergeat V. Recent advances in the transcriptional regulation of the flavonoid biosynthetic pathway. J Exp Bot. 2011;62:2465-83.

40. Honda C, Moriya S. Anthocyanin biosynthesis in apple fruit. Hortic J. 2018; 87:305-14.

41. Allan AC, Hellens RP, Laing WA. MYB transcription factors that color our fruit. Trends Plant Sci. 2008;13:99-102.

42. Takos AM, Jaffé FW, Jacob SR, Bogs J, Robinson SP, Walker AR. Lightinduced expression of a MYB gene regulates anthocyanin biosynthesis in red apples. Plant Physiol. 2006;142:1216-32.

43. Espley RV, Hellens RP, Putterill J, Stevenson DE, Kutty-Amma S, Allan AC. Red colouration in apple fruit is due to the activity of the MYB transcription factor, MdMYB10. Plant J. 2007:49:414-27.

44. Wang XF, An JP, Liu X, Su L, You CX, Hao YJ. The nitrate-responsive protein MdBT2 regulates anthocyanin biosynthesis by interacting with the MdMYB1 transcription factor. Plant Physiol. 2018;178:890-906.

45. An JP, Yao JF, Xu RR, You CX, Wang XF, Hao YJ. Apple bZIP transcription factor MdbZIP44 regulates abscisic acid-promoted anthocyanin biosynthesis. Plant Cell Environ. 2018:41:2678-92.

46. An JP, Zhang XW, You CX, Bi SQ, Wang XF, Hao YJ. MdWRKY40 promotes wounding-induced anthocyanin biosynthesis in association with MdMYB1 and undergoes MdBT2-mediated degradation. New Phytol. 2019;224:380-95.

47. An JP, Liu YJ, Zhang XW, Bi SQ, Wang XF, You CX, Hao YJ. Dynamic regulation of anthocyanin biosynthesis at different light intensities by the BT2-TCP46-MYB1 module in apple. J Exp Bot. 2020;71(10):3094-109.

48. An JP, Wang XF, Hao YJ. BTB/TAZ protein MdBT2 integrates multiple hormonal and environmental signals to regulate anthocyanin biosynthesis in apple. J Integr Plant Biol. 2020;62(11):1643-6.

49. An JP, Zhang XW, Bi SQ, You CX, Wang XF, Hao YJ. The ERF transcription factor MdERF38 promotes drought stress-induced anthocyanin biosynthesis in apple. Plant J. 2020;101:573-89.

50. Li YY, Mao K, Zhao C, Zhao XY, Zhang HL, Shu HR, Hao YJ. MdCOP1 ubiquitin E3 ligases interact with MdMYB1 to regulate light-induced anthocyanin biosynthesis and red fruit coloration in apple. Plant Physiol. 2012;160(2):1011-22.

51. Hao YJ, Kitashiba H, Honda C, Nada K, Moriguchi T. Expression of arginine decarboxylase and ornithine decarboxylase genes in apple cells and stressed shoots. J Exp Bot. 2005;56(414):1105-15.

52. Kang H, Zhang TT, Fu LL, You CX, Wang XF, Hao YJ. The apple RING-H2 protein MdCIP8 regulates anthocyanin accumulation and hypocotyl elongation by interacting with MdCOP1. Plant Sci. 2020;301:110665.

53. Dai H, Li W, Han G, Yang Y, Ma Y, Li H, Zhang Z. Development of a seedling clone with high regeneration capacity and susceptibility to agrobacterium in apple. Sci Hortic. 2013;164:202-8.

54. Geng DL, Chen PX, Shen XX, Zhang Y, Li XW, Jiang LJ, Xie YP, Niu CD, Zhang J, Huang XH, Ma FW, Guan QM. MdMYB88 and MdMYB124 enhance drought tolerance by modulating root vessels and cell walls in apple. Plant Physiol. 2018;178(3):1296-309.

55. Zhang YL, Zhang CL, Wang GL, Wang YX, Qi CH, Zhao Q, You CX, Li YY, Hao YJ. The R2R3 MYB transcription factor MdMYB30 modulates plant resistance against pathogens by regulating cuticular wax biosynthesis. BMC Plant Biol. 2019;19(1):362.

56. Clough SJ, Bent AF. Floral dip: a simplified method for agrobacteriummediated transformation of Arabidopsis thaliana. Plant J. 1998;16(6):735-43.

57. Xie XB, Li S, Zhang RF, Zhao J, Chen YC, Zhao Q, Hao YJ. The bHLH transcription factor MdbHLH3 promotes anthocyanin biosynthesis and fruit colouration in response to low temperature in apples. Plant Cell Environ. 2012;35(11):1884-97. 
58. Zhang CL, Mao K, Zhou LJ, Wang GL, Zhang YL, Li YY, Hao YJ. Genomewide identification and characterization of apple long-chain acyl-CoA synthetases and expression analysis under different stresses. Plant Physiol Biochem. 2018;132:320-32.

59. Qi CH, Jiang H, Zhao XY, Mao K, Liu HT, Li YY, Hao YJ. The characterization, authentication, and gene expression pattern of the MdCER family in Malus domestica. Horticultural Plant Journal (HPJ). 2018;5(1):1-9.

60. Ren YR, Yang YY, Zhang R, You CX, Zhao Q, Hao YJ. MdGRF11, an apple 143-3 protein, acts as a positive regulator of drought and salt tolerance. Plant Sci. 2019;288:110219.

61. Lee HS, Wicker L. Anthocyanin pigments in the skin of lychee fruit. J Food Sci. 1991;56:466-8.

62. An JP, Wang XF, Zhang XW, Xu HF, Bi SQ, You CX, Hao YJ. An apple MYB transcription factor regulates cold tolerance and anthocyanin biosynthesis and undergoes MIEL1-mediated degradation. Plant Biotechnol J. 2020;18(2): 337-53.

\section{Publisher's Note}

Springer Nature remains neutral with regard to jurisdictional claims in published maps and institutional affiliations.

Ready to submit your research? Choose BMC and benefit from:

- fast, convenient online submission

- thorough peer review by experienced researchers in your field

- rapid publication on acceptance

- support for research data, including large and complex data types

- gold Open Access which fosters wider collaboration and increased citations

- maximum visibility for your research: over $100 \mathrm{M}$ website views per year

At BMC, research is always in progress.

Learn more biomedcentral.com/submissions 